\title{
Nursing clinical trial of breast self-examination education in China
}

\section{C.-Y. Liu' MD, H.-O. Xia ${ }^{2}$ RN, PhD, D.M. Isaman ${ }^{3} \mathrm{PhD}$, W. Deng ${ }^{4,5}$ RN, GNP, MS \& D. Oakley ${ }^{6}$ PhD}

I Professor and Dean Emeritus, School of Nursing, Tianjin Medical University, Tianjin, 2 Associate Professor, School of Nursing, FuDan University, Shanghai, China, 3 Assistant Research Scientist, 4 Research Associate, 6 Professor Emerita, School of Nursing, University of Michigan, Ann Arbor, MI, 5 Gerontological Nurse Practitioner, Onlok, Fremont, CA, USA

LIU C.-Y., XIA H.-O., ISAMAN D.M., DENG W. \& OAKLEY D. (2010) Nursing clinical trial of breast self-examination education in China. International Nursing Review 57, 128-134

Aim: The aim of this study was to test community-based nursing education about breast self-examination to see whether self-examination frequency could be increased.

Background: Breast cancer rates in China are rising rapidly, especially in cities. The majority of Chinese women does not know about breast self-examination, and the Chinese health care system cannot provide mammograms for the millions of at-risk women throughout China.

Method: This study was a randomized clinical trial of nurse-provided, community-based teaching of breast self-examination in the urban and rural areas of Tianjin and urban and suburban areas of Shanghai. Women ( $n=1510)$ never diagnosed with breast cancer and 40 years and older were randomized by community and stratified by urban vs. other residences.

Results: At baseline, $9 \%$ of the intervention and $6 \%$ of the control groups did breast self-examination at least every other month. After 12 months, 34\% of the intervention, but only $11 \%$ of the control, group did breast self-examination that often $(P<0.001)$. There was a significant impact in urban, suburban and rural areas, and intervention effects were stronger than any of the other influences tested.

Conclusion: The clinical trial showed a statistically significant increase in breast self-examination after the nursing education intervention. As no other breast cancer screening method is available in most of China, this method is best suited for the masses of people currently at risk in China.

Keywords: Breast Cancer, Breast Self-Examination, China, Community, Health Education, Nursing

\section{Introduction}

China faces an alarming increase in breast cancer rates, largely because of an increase in risk factors associated with changing lifestyles (Yang et al. 2005). For women 45-49 years, prevalence was less than 100 per 100000 in 1999 but is projected to reach 121 in 2010 and 145 per 100000 by 2020 (Zhen et al. 2001).

Correspondence address: Deborah Oakley, University of Michigan School of Nursing: GRO, 400 N. Ingalls, Ann Arbor, MI 48109-5482, USA; Tel: 1-734-763-6730; Fax: 1-734-615-1666; E-mail: doakley@umich.edu.
Breast is now the most frequently diagnosed cancer in Shanghai, Beijing, Tianjin and Guangzhou (Gao 1999; Hao et al. 2002; Li 2004; People's Daily 2003a; Yang et al. 2004; Ying \& Zhang 2003). By 2021, 2.5 million women aged 35-49 years in 2001 are expected to have breast cancer. China is 'on the cusp of a breast cancer epidemic' (Linos et al. 2008).

In nations with adequate medical resources, mammograms are the standard screening technique for early detection. In China, mammograms are available only at a limited number of urban hospitals. The Chinese government policy is recognized as the foundation for change, but there is no mandate to provide 
free or low-cost mammograms on the mass scale required. In most cases, the cost must be paid by the individual (Wong \& Gabriel 2000). Recognizing the situation in many low-resource countries, the World Health Organization (WHO) continues to recommend breast self-examination (BSE) until more technologically advanced alternatives are available (World Health Organization 2002). The WHO's support for BSE is based on its estimate that two-thirds of all cancers, including breast cancer, can be prevented or treated successfully if detected early (World Health Organization 2005). Its recommendation is based on research showing that BSE can be associated with finding breast cancer at a more favourable clinical stage (Foster et al. 1978).

The study reported here started at the first step in examining the effectiveness of BSE education by asking the following questions:

1 Does BSE increase after a BSE educational intervention is delivered in communities of residence by nurses in China?

2 After the intervention, does BSE increase among the women living in both urban and non-urban areas?

3 In addition to the intervention, do women's demographic and/or psychosocial characteristics help predict more frequent BSE?

\section{Methods}

\section{Setting}

Study sites were urban and suburban neighbourhoods within the city of Shanghai and urban neighbourhoods and rural communities within the official city boundaries of Tianjin. Because it is possible that city residents are more likely to adopt communitybased innovations, community randomization was stratified to include urban, suburban and rural residents. The neighbourhoods selected were those whose leaders were willing to collaborate with the two schools of nursing. In China, access to neighbourhoods is still through leaders designated by the local authorities. Once the available urban and non-urban neighbourhoods were identified in both Shanghai and Tianjin, they were randomly assigned to treatment or control using random number generation, so that for each type of area there were both treatment and control communities. This type of cluster randomization was used to minimize treatment contamination as numerous women would be living in the same apartment buildings, or otherwise, in close proximity.

During the baseline group session, Chinese-language consent forms that had been approved by the University of Michigan Institutional Review Board for ethical research were read to all women and signed in person. Personal information to facilitate re-contact was separated from the questionnaires, and study files were maintained using study identification numbers only.

\section{Participants}

All women who were 40 years of age and older, and who had never been diagnosed with breast cancer were asked to participate. A total of 1510 women were recruited, 739 in Shanghai (360 in urban and 379 in suburban neighbourhoods) and 771 in Tianjin (381 in urban neighbourhoods and 390 in rural communities). Every participant was asked to return for the 12-month follow-up; those who did not were sought for telephone or home interview. A total of $91 \%$ of the urban and $90 \%$ of the non-urban participants could be reached for follow-up, proportions that enhance validity (Scott 2004). It is relevant that our communitybased design facilitated continued contacts. Those reached for follow-up did not differ significantly, in demographic or social characteristics, from the total in each of the subgroups at recruitment. During the study period, four women were found to have breast cancer.

\section{Intervention and control groups}

BSE is most effective when performed carefully and regularly (Fletcher et al. 1990). Therefore, nursing faculty and graduate students were systematically trained for the study in bilingual group settings during December 2005. For the intervention group, the certified Mammacare nurse team member used Mandarin for training following Mammacare techniques. The Mandarin-language Mammacare video was used. (This video is available from http://www.mammacare.com. With their permission, we converted the video into numerous digital video discs (DVDs) as the video systems are different in the USA and China.) A PowerPoint lecture about breast cancer, the importance of early screening and changing rates in China was translated into Mandarin and video recorded at the Shanghai site. Mammacare breast models were used for practice and testing. The whole process was repeated in Tianjin. During 2006, faculty and graduate students from Tianjin Medical University and FuDan University Schools of Nursing used all of these same protocols and materials in their workshops with the intervention group community residents. After the 1-h seminar, practice and testing, intervention group participants were given a specially designed take-home BSE brochure. This brochure was the product of earlier focus groups and pre-tests with women 40+ years of age at the two universities in China. Intervention group women also received a Mandarin-language 'hang' tag to record monthly BSE. (This hang tag is available from the Susan G. Komen Breast Cancer Foundation.)

For the control group, the same faculty and students used protocols and materials selected by the research team at team meetings earlier in 2005 in Ann Arbor and Shanghai. They taught about knowledge and behaviour needed to maintain health and prevent disease, following principles of equivalence 
and attractiveness (Lindquist et al. 2007). During the controlgroup sessions, they mentioned other wellness activities, including prevention of diabetes mellitus, back pain and various kinds of cancer, but did not give specific instructions or encouragement. The sessions lasted about $1 \mathrm{~h}$. There was no take-home information. At the end of the study, control-group members were offered the intervention's educational seminar and takehome materials.

\section{Questionnaires}

At baseline, all participants completed a 101-item questionnaire about breast cancer screening behaviours, knowledge and attitudes; access and barriers to care; breast cancer risk and perceived susceptibility; perceived severity of breast cancer; social support; and information sources about breast cancer. The screening frequency variable was measured as $1=$ never, $2=$ not regularly, $3=$ every 2 months and $4=$ every month. Almost all items were check-offs, and completing them took about $20 \mathrm{~min}$. Satisfactory psychometric qualities of all items and scales developed from them had been established in prior studies (Yu et al. 2005) and in the 2005 pilot work at each of the study sites.

Twelve months later, to evaluate the effects of the intervention, the items about screening behaviours, breast cancer knowledge, attitude towards screening, perceived susceptibility, perceived severity and perceived benefits of screening, and barriers to screening were repeated.

\section{Analysis}

Statistical results used data entered into the SPSS 16.0 (SPSS, Inc., Chicago, IL, USA) using templates established before data collection and reviewed after data entry at the University of Michigan, with consistency checks for outliers and erroneous entries. Also, checks for internal consistency over time were conducted for questions repeated at follow-up. Scales were created by counting the 'yes' answers for access, counting the correct answers for knowledge and averaging the agree-to-disagree scale for attitude, social support and mastery items. When appropriate, variables were reverse-coded before aggregation.

Our primary analysis was testing the difference between the intervention and the control groups. First, we compared the two groups at baseline for important demographic and risk variables using a difference of proportions for dichotomous variables. In particular, we tested self-reported performance of BSE at baseline (yes/no). We then did the identical test of proportions for performance of BSE at 12 months follow-up.

To adjust for both baseline performance of BSE and site differences, we used the analysis of variance to test the differences between groups. The outcome variable was a difference score of the frequency of BSE at 12 months follow-up minus the frequency of BSE at baseline. The differencing of these fourpoint ordinal scales makes the difference score less discrete. Multiple comparisons for significant F-tests were performed using Tukey's pairwise comparisons. Finally, tests of assumptions (including normality) were performed.

To determine whether other predictors might also affect BSE frequency, we used a two-stage approach with multiple linear regression. In the first stage, backward regression was performed separately for sets of demographic, health and multi-item scales. Those that were significant at $P<0.10$ were retained and, in the second stage, again subjected to backward regression using $\alpha=0.05$. Further exploration of the main result was pursued using the same approach and descriptive comparison of the amount of change from baseline. Baseline values were used as the most appropriate predictors of the subsequent behaviours during the study period.

\section{Results}

At baseline, the intervention and control groups did not differ on any of the characteristics measured, as shown in Table 1. On average, the women were middle-aged, had completed high school or less, were married and shared a living space with no or only one other person. They were also similar in other respects: about one-third had learned about BSE before, but only a few did BSE every month or every other month, or said they felt complete mastery of doing BSE. Two-fifths of both groups said they got their breast screening information from the television. In addition, $7 \%$ of both groups reported having a family member with a breast cancer diagnosis. Half of both groups thought early detection was important, and the two groups had the same moderate breast cancer knowledge, generally positive attitudes and tendency to discuss breast cancer among friends and relatives.

Table 1 Characteristics of study participants at baseline

\begin{tabular}{llc}
\hline Characteristics & Intervention & Control \\
\hline & & \\
Mean age & 53.7 years & 49.9 years \\
High school education or less & $76.3 \%$ & $83.6 \%$ \\
Married & $91.0 \%$ & $92.7 \%$ \\
Sharing living space with 3+ people & $11.3 \%$ & $11.5 \%$ \\
Learned BSE before study & $31.2 \%$ & $35.4 \%$ \\
Did BSE every month or two & $8.5 \%$ & $5.9 \%$ \\
Got breast cancer screening information & & \\
$\quad$ Via the television & $40.6 \%$ & $41.8 \%$ \\
$\quad$ Via newspapers & $37.3 \%$ & $37.9 \%$ \\
\end{tabular}

$P \geq 0.10$, i.e. no significant differences between intervention and control groups.

BSE, breast self-examination. 
For instance, about three-quarters of both groups said they talked about breast cancer with their husbands, friends and doctors.

A 12-month data showed that those in the intervention groups were much more likely to know about BSE: 99 vs. $59 \%$ for the control groups $(P<0.001)$. Most importantly, the intervention had a statistically significant $(P<0.001)$ effect, increasing selfreported regular (monthly or every 2 months) BSE from $9 \%$ at baseline to $34 \%$ during the study period among the intervention group compared with the time-dependent increase from $6 \%$ to just $11 \%$ among the control group. The moderate intervention effects are well within the range typically found in health behaviour studies (Hall \& Rossi 2008). Further, the BSE educational intervention made a statistically significant difference (improvement) in the frequency of BSE, in both locations (Shanghai and Tianjin), and no matter whether the women lived in urban or non-urban areas. Notably, these statistically significant intervention effects occurred for each study site while most of the control communities drifted upward in the reported BSE frequency. This general drift may be related to some local efforts, during this period, to promote knowledge about the importance of, and techniques for, early detection of breast cancer (People's Daily 2002, 2003b).

To determine whether other predictors might also affect BSE frequency, we used a two-stage approach with multiple linear regression (Zar 1999). In the first stage, backward regression was performed separately for the sets of demographic (marital status, education, parity, cohabitation and age), health (health status, insurance, accessibility of providers, time availability, availability of screening and affordability of screening) and multi-item scales (knowledge, perceived benefit, perceived risk and perceived barriers). The site and intervention status, and the interaction between these two, were also included. Those that were significant at $P<0.10$ were retained and, in the second stage, again subjected to backward regression using $\alpha=0.05$. In the final regression model, as indicated in Table 2, only the intervention, perceived barriers at follow-up and mastery at follow-up had effects on increasing the frequency of BSE (adjusted $R^{2}=0.256$ ). The interaction term for location $\times$ intervention (or not) was also statistically significant because greater increases in frequency occurred in Shanghai's urban, but Tianjin's rural areas, perhaps because Shanghai's suburban residents and Tianjin's urban residents were similar. The intervention alone had an adjusted $R^{2}=0.251$, thus accounting for the vast majority (98\%) of the explained variance.

Further exploration of the barrier scale indicated that baseline to outcome changes in a few items were particularly affected. As shown in Table 3, these four items were perceptions about doctors and males, ability to do BSE and fear of mammograms. For each item, the changes were greatest in the middle of the scales, moving from more to less emphasis on the item as a barrier. Percentage distributions are shown for the total sample because the barrier scale had a significant influence on change in the frequency of BSE during the period, independent of the intervention group. These results were the same in each study site (data not shown). The four items were selected because, of the 20 total, these were the most influential. It is culturally relevant to note that, at baseline, more than half of the participants in each of the four types of study sites (city $\times$ urban status) said they see doctors only when they are sick.

Multiple regression analysis (not shown) suggests that perceived mastery is a more complex phenomenon. In an analysis

Table 2 Predictors of change in the frequency of breast self-examination (BSE)

\begin{tabular}{|c|c|c|c|c|c|}
\hline Predictor & Sum of squares & $d f$ & Mean square & $F$ & Significance \\
\hline Corrected model & 462.459 & 9 & 51.384 & 53.053 & 0.000 \\
\hline Intercept & 79.325 & 1 & 79.325 & 81.901 & 0.000 \\
\hline Intervention & 57.137 & 1 & 57.137 & 58.992 & 0.000 \\
\hline Site: urban/non-urban & 30.099 & 3 & 10.033 & 10.359 & 0.000 \\
\hline Perceived barriers & 38.151 & 1 & 38.151 & 39.390 & 0.000 \\
\hline Perceived mastery of BSE & 171.707 & 1 & 171.707 & 177.282 & 0.000 \\
\hline \multicolumn{6}{|l|}{ Interaction } \\
\hline Intervention $\times$ site & 13.644 & 3 & 4.548 & 4.696 & 0.003 \\
\hline Error & 1308.516 & 1351 & 0.969 & & \\
\hline Total & 2218.000 & 1361 & & & \\
\hline Corrected total & 1770.976 & 1360 & & & \\
\hline
\end{tabular}

Adjusted $R^{2}=0.256$.

$d f$, degree of freedom. 
Table 3 Percentage baseline and outcome responses to selected barriers

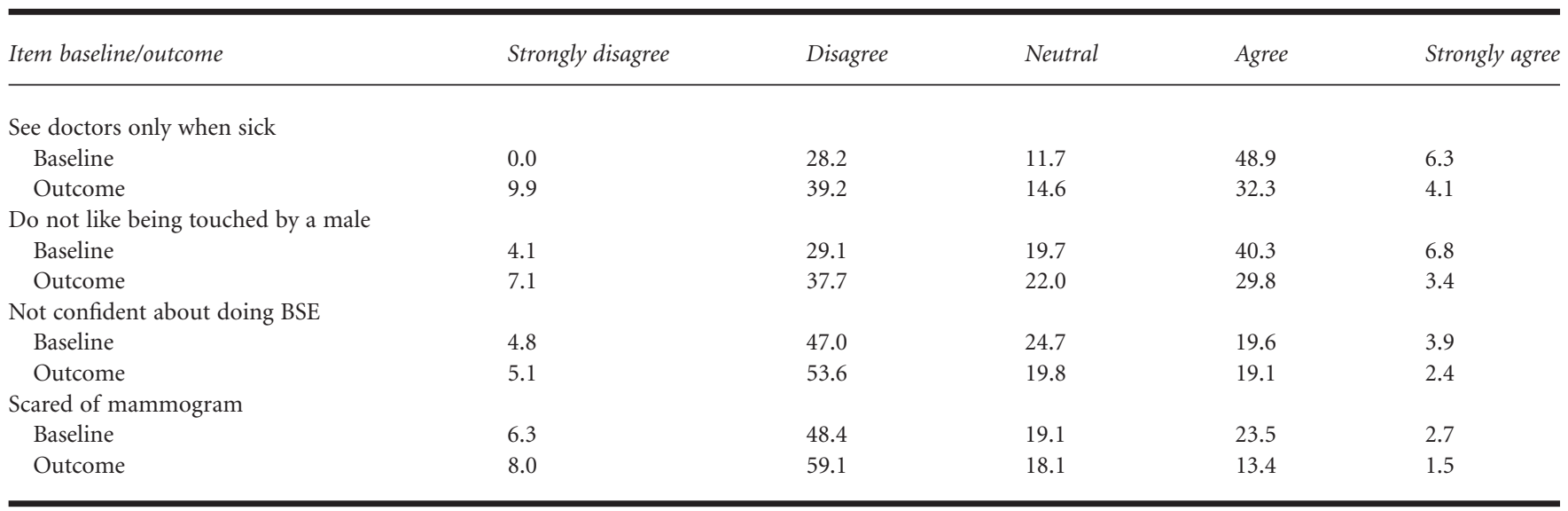

BSE, breast self-examination.

using change in BSE mastery as the dependent variable, more variables were associated with an increasing sense of mastery. Availability of providers, a measure of health care access, was highly significant. Attitude, perceived severity of breast cancer, perceived barriers, knowledge and the interaction term of city $\times$ urban status also made significant independent contributions to predicting higher increases in BSE mastery, but overall, the explained variance was relatively low, with adjusted $R^{2}=0.185$.

\section{Discussion}

Our findings support the validity of women's responses in this study, as behavioural theories predict that (1) interventions will affect the middle of a distribution before the extremes and that (2) the amount of change will be moderate. One lesson is that interventions may be most effective if targeted particularly towards those with less strong feelings about the most common barriers. Another lesson is that the intervention may need to emphasize mastery of doing one's own breast examination. Hands-on teaching that includes practice, such as the Mammacare technique for BSE, is an effective way to increase the sense of mastery. Our results suggest that even one educational session, augmented by take-home informational materials and very brief quarterly contacts, can suffice.

According to these results, it is possible to increase the frequency of BSE practice with a standardized educational, community-based programme delivered by nurses. Importantly, these effects can be obtained in non-urban as well as urbanized areas in both northern and middle China. Thus, the response to the first two research questions is positive: yes, it is possible to increase BSE frequency, and the same programme can produce results in urban and non-urban areas.
We have also shown that among these women who are 40 years and older, age, education, marital status or cohabitation, health status, knowledge about breast cancer, perceived risk from breast cancer and time availability did not directly add to the intervention's effect on increasing BSE frequency. However, lower scores on the scale of perceived barriers had a small, yet independent, effect on increasing BSE frequency. The major barriers of reluctance to see a doctor until sick, not liking to see a male doctor, not feeling confident about doing BSE and being scared of having a mammogram are all related to culture (Choe et al. 2006; Sun et al. 2004; Wang et al. 2006) and probably also to prior lack of available, easily accessible care. In summary, the intervention increases BSE frequency among a wide variety of women. As accessibility increases, and modernization spreads, cultural barriers may also be reduced.

Until now, limited health care resources have been unable to support prevention and early detection in China. Now that the government has undertaken large-scale public education programmes to promote knowledge about the importance of pre-symptom prevention, BSE is ideal, as it requires only the intervention educators and is well-suited to China's communitybased living conditions. At the least, BSE makes women personally aware of the individual relevance of prevention.

The Chinese medical system often reaches out through district hospitals, where staff are sent to neighbourhoods and/or outlying areas to conduct health campaigns (Cheung 1995; People's Daily Online, 2006; Xiangyang et al. 2003). Early detection through BSE can be combined with dietary recommendations that may help reduce projected increases in breast cancer (Linos et al. 2008). As the alarming increases in breast cancer in China have been shown to be mainly a result of an increase in risk factors (Leung et al. 2002; Yang et al. 2004), an educational 
intervention that teaches BSE and promotes avoidance or reduction of risk behaviours is timely, if not imperative. Higher dietary fat and smoking are known risk factors for breast cancer in the Chinese population (Yuan et al. 1995; Zhao et al. 1999) and typically increase as rural populations move into urban areas (Gardner et al. 2002). As China's urban population has increased from $20 \%$ to about $40 \%$ of the total and is still rising (Riley 2004), risk factors will affect more and more people. Even urbanized migrants who return to their rural homes during economic downturns will spread new tastes and practices.

Prevention is a key idea that can include, even start with, BSE education. Three prongs can be suggested: (1) incorporating BSE into all nursing school curricula, (2) sending nursing, public health and/or district hospital teams to urban, suburban and rural health centres to conduct annual or semi-annual half-day BSE education sessions, and (3) making televised and internet BSE education modules (Im \& Chee 2008) available, followed by monthly reminders to do BSE.

China is not an isolated case among Asian populations (Lee et al.2008), although the population at risk is many times higher than in most other emerging nations. China can be a model for containing the increase in breast cancer mortality as modernization spreads. Health professionals in China have the resources and experience to conduct the campaigns required. The first step is to develop guidelines at the Ministry of Health level and to disseminate these to regions and to professional organizations. The next step is to oversee implementation, including process, outcome and impact evaluation.

\section{Study limitations}

The well-known earlier 'Shanghai study' of BSE showed effectiveness in earlier detection but not in mortality reduction (Gao et al. 2005; Thomas et al. 2002). Thus, we cannot be certain that BSE education will reduce mortality throughout China. However, we should note that the Shanghai study results were affected by history. Even the study authors acknowledged that the 5-year follow-up period saw economic reorganization with turmoil among some of the participating factories. Access to medical care could have been curtailed, affecting women's ability to follow through on early detection (Davis \& Chapman 2002; Hu 2004; Wong \& Gabriel 2000). Pilot reform projects to restore more adequate health insurance did not even begin until 1994 (Liu et al. 2007). In any case, BSE is the only realistic screening technique available in China, as in other low-resource countries (World Health Organization 2002). Further studies are needed to confirm that BSE education is feasible and effective for populations living farther from cities with communities that are officially rural but within urban-area commuting distance.

\section{Acknowledgement}

This work was supported by the Susan G. Komen Breast Cancer Foundation (grant no. POP0503380), made originally to the late Mei-yu Yu (1944-2005).

\section{Author contributions}

C.-Y. Liu is the co-first author and was involved in the study design, site supervision, intervention design and delivery, data collection and entry supervision, and manuscript revisions, and provided administrative support. H.-O. Xia is the co-first author and was involved in the study conception, site supervision, data collection and entry supervision, and manuscript revisions, and provided administrative support. D.M. Isaman was involved in the study design, data template design, statistical analysis, manuscript preparation and revisions. W. Deng was involved in the study design, data collection and intervention training, and manuscript revisions, and acted as bilingual-bicultural advisor. D. Oakley was involved in the study design, data collection training, manuscript preparation and revisions, and provided administrative support as principal investigator.

\section{References}

Cheung, Y.B. (1995) Community mobilization and health care in rural china. Community Development Journal, 30, 317-326.

Choe, J.H., et al. (2006) 'Heat in their intestine': colorectal cancer prevention beliefs among older Chinese Americans. Ethnicity \& Disease, 16, 248-254.

Davis, D. \& Chapman, N.E. (2002) Turning points in Chinese health care: crisis or opportunity? Yale-China Health Journal, 1 (Autumn), 3-9.

Fletcher, S.W., et al. (1990) How best to teach women breast selfexamination: a randomized controlled trial. Annals of Internal Medicine, 112, 772-779.

Foster, R.S., et al. (1978) Breast self-examination practices and breastcancer stage. The New England Journal of Medicine, 299, 2665-2670.

Gao, D.L. (1999) The evaluation of etiology and prevention of breast cancer [in Chinese]. Zhang Guo Lin Chuang Yi Xue Za Zhi [Clinical Medicine Journal of China], 6, 319-320.

Gao, D.L., et al. (2005) Randomized trial of breast self-examination in 266,064 women in Shanghai [in Chinese]. Zhonghua Zhong Liu Za Zhi [Chinese Journal of Oncology], 27, 350-354.

Gardner, K.M., et al. (2002) Occupations and breast cancer risk among Chinese women in urban Shanghai. American Journal of Industrial Medicine, 42, 296-308.

Hall, K.L. \& Rossi, J.S. (2008) Meta-analytic examination of the strong and weak principles across 48 health behaviors. Preventive Medicine, 46 , 266-274.

Hao, X.S., Chen, K.S. \& Liu, H. (2002) Analysis of female breast cancer descriptive epidemiology in Tianjin, China. Asian Pacific Journal of Cancer Prevention, 3, 143-148.

Hu, T.W. (2004) Financing and organization of China's health care. Bulletin of the World Health Organization, 82, 480. 
Im, E.-O. \& Chee, W. (2008) The use of internet cancer support groups by ethnic minorities. Journal of Transcultural Nursing, 19 (1), 74-82.

Lee, S.-M., Park, J.-H. \& Park, H.-J. (2008) Breast cancer risk factors in Korean women: a literature review. International Nursing Review, 55, 355-359.

Leung, G.M., et al. (2002) Trends in breast cancer incidence in Hong Kong between 1973 and 1999: an age-period-cohort analysis. British Journal of Cancer, 87, 982-988.

Li, Y.H. (2004) Breast cancer in big cities of China. Greater Detroit ChineseAmerican News, 7 May, 7.

Lindquist, R., et al. (2007) Design of control-group conditions in clinical trials of behavioral interventions. Journal of Nursing Scholarship, 39, 214-221.

Linos, E., et al. (2008) Effects of reproductive and demographic changes on breast cancer incidence in China: a modeling analysis. Journal of the National Cancer Insitute, 100, 1352-1360.

Liu, G.G., et al. (2007) Urban health care reform initiative in China: findings from its pilot experiment in Zhenjiang City. International Journal of Economic Development, 1, 504-522.

People's Daily (2002) China, US raise breast cancer awareness in China: the Chinese People's Association for Friendship with Foreign Countries (CPAFFC) and People to People International (PTPI) of the United States jointly launched a breast cancer awareness project for China Wednesday. Available at: http://english.peopledaily.com.cn/200203/28/ eng20020328_92965.shtml (accessed 24 September 2007).

People's Daily (2003a) Increasing urban health problems. Available at: http://www.english.peopledaily.com.cn/200309/01/ eng20030901_123472.shtml (accessed 26 September 2007).

People's Daily (2003b) China launches campaign against breast cancer: China's health authorities are launching a massive campaign against breast cancer, which has grown to become the biggest killer of Chinese women. Available at: http://english.peopledaily.com.cn/200309/01/ eng20030901_123472.shtml (accessed 24 September 2007).

People's Daily Online (2006) China issues policies to launch 'new socialist countryside’. Available at: http://english.people.com.cn/200602/21/ eng20060221_244642.html (accessed 24 September 2007).

Riley, N.E. (2004) China's population: new trends and challenges. Population Bulletin, 59 (2), 1-40.

Scott, C.K. (2004) A replicable model for achieving over 90\% follow-up rates in longitudinal studies of substance abusers. Drug and Alcohol Dependence, 74, 21-36.
Sun, W.Y., Basch, C.E., Wolf, R.L. \& Li, X.J. (2004) Factors associated with colorectal cancer screening among Chinese-Americans. Preventive Medicine, 39, 323-329.

Thomas, D.B., et al. (2002) Randomized trial of breast self-examination in Shanghai: final results. Journal of the National Cancer Institute, 94, 14451457.

Wang, J.H.Y., et al. (2006) The influence of culture and cancer worry on colon cancer screening among older Chinese-American women. Ethnicity \& Disease, 16, 404-411.

Wong, B.A. \& Gabriel, S.J. (2000) The influence of economic liberalization on urban health care access in the People's Republic of China. Available at: http://cob.mtholyoke.edu/courses/sgabriel/chinahealth.htm (accessed 12 December 2000).

World Health Organization (2002) National Cancer Control Programmes: Policies and Managerial Guidelines. WHO, Geneva, Switzerland.

World Health Organization (2005) WHO Cancer Control Programme. Available at: http://www.who.International (accessed 25 October 2008).

Xiangyang, T., et al. (2003) Beijing health promoting universities: practice and evaluation. Health Promotion International, 18, 107-113.

Yang, L., et al. (2004) Estimation and projection of the national profile of cancer mortality in China: 1991-2005. British Journal of Cancer, 90, 2157-2166.

Yang, L., et al. (2005) Estimates of cancer incidence in China for 2000 and projections for 2005. Cancer Epidemiology, Biomarkers \& Prevention, 14, 243-250.

Ying, H. \& Zhang, Z.X. (2003) Breast cancer incidence rate increased 40\% in Tianjin. Available at: http://athletic.eastday.com (accessed 4 August 2004).

Yu, M.Y., Wu, T.Y. \& Mood, D.M. (2005) Cultural affiliation and mammography screening of Chinese women in an urban county of Michigan. Journal of Transcultural Nursing, 16 (2), 107-116.

Yuan, J.M., et al. (1995) Diet and breast cancer in Shanghai and Tianjin, China. British Journal of Cancer, 71, 1353-1358.

Zar, J.H. (1999) Biostatistical Analysis, 4th edn. Prentice-Hall, Inc., Upper Saddle River, NJ.

Zhao, Y., Shi, Z. \& Liu, L. (1999) Matched case-control study for detecting risk factors of breast cancer in women living in Chengdu [in Chinese]. Chung-Hua Liu Hsing Ping Hsueh Ts Chic [Chinese Journal of Epidemiology], 20 (2), 91-94.

Zhen, Y., et al. (2001) An analysis of breast cancer incidence rate in Shanghai [in Chinese]. Wai Ke Li Lun Yu Shi Jian [Surgical Theory \& Practice], 6, 219-221. 